\title{
A Custom-Made Modular Mega-Spacer for the Treatment of Infection of a Total Femur Prosthesis: A New Surgical Technique
}

\author{
Bernhard M. Speth, Andreas H. Krieg, Fritz Hefti, and Andrej M. Nowakowski ${ }^{2}$
}

\begin{abstract}
Background: Limb salvage surgery (LSS) with endoprosthetic replacement is a common method of reconstruction after bone tumor resection whenever there is no possibility for a biologic reconstruction. Postoperative infection is the most common reason for failure. Two-stage procedures require spacers to maintain the distance and to allow for mobilization. Because of the rarity and diversity of these cases there are no commercially available pre-fabricated solutions.

Case Presentation: We present a simple novel method for a custom-made antibiotic-coated mega-spacer that we applied successfully in a patient with an infected mega-endoprosthesis after a wide resection of an osteosarcoma. Conclusion: Through its modularity the illustrated technique offers a good and inexpensive solution for individual adjustments for the preparation of interim prostheses especially for two-stage exchanges of megaendoprosthesis. The optimum length can easily be adjusted. Furthermore, the explanation of the mega-spacer before replacement of a final prosthesis is simplified.
\end{abstract}

Keywords: mega-spacer; prosthetic-joint infection; spacer; total femur; two-stage treatment

$\mathbf{L}$ IMB SALVAGE SURGERY (LSS) with endoprosthetic replacement is a common method of reconstruction after bone tumor resection whenever there is no possibility of a biologic reconstruction [1]. Post-operative infection is the most common reason for failure [2] for tumor endoprosthesis and the risk for it is high $(10 \%)[3,4]$ compared with conventional arthroplasty. In oncologic patients the susceptibility for infection is increased through pre-operative chemotherapy or radiotherapy.

Two-stage procedures require spacers to maintain the distance and to allow for mobilization [5]. The problem in substituting femoral implants with a spacer is the need for an individual spacer $[5,6]$.

We present a simple method for a custom-made antibioticcoated mega-spacer that we applied successfully in a patient with an infected mega-endoprosthesis after a wide resection of an osteosarcoma. The method is simple and inexpensive and offers the possibility for individual solutions.

\section{Case Presentation and Surgical Technique}

A 13-year-old patient with high-grade osteoblastic osteosarcoma of the femur with involvement of the proximal ephysis underwent a wide resection and implantation of a total femur endoprosthesis after neoadjuvant chemotherapy (Fig. 1). During the post-operative course and adjuvant chemotherapy the patient developed a deep implant-associated infection with coagulase-negative staphylococci (CoNS) and Klebsiella pneumonia. The infection together with a septic shock determined the indication for the two-step revision of the implant according to the algorithm described by Zimmerli et al. [7]. After removal of the prosthesis and of the acetabular cartilage we implanted an individualized mega-spacer. The design for the spacer was developed by two of the authors (F.H. and A.M.N.) and surgery was performed by the senior author (A.M.N). The head was made with a cement spacer mold (Stage one select R, Biomet, Warsaw, IN) and coupled with a 6-mm Harrington stainless steel rod. Under traction, the

\footnotetext{
${ }^{1}$ Department of Pediatric Orthopedic Surgery, University Children's Hospital Basel, Basel, Switzerland.

${ }^{2}$ Orthopedic Department, University Hospital Basel, University of Basel, Basel, Switzerland.

(C) Bernhard Speth et al. 2016; Published by Mary Ann Liebert, Inc. This Open Access article is distributed under the terms of the Creative Commons License (http://creativecommons.org/licenses/by/4.0), which permits unrestricted use, distribution, and reproduction in any medium, provided the original work is properly credited.
} 


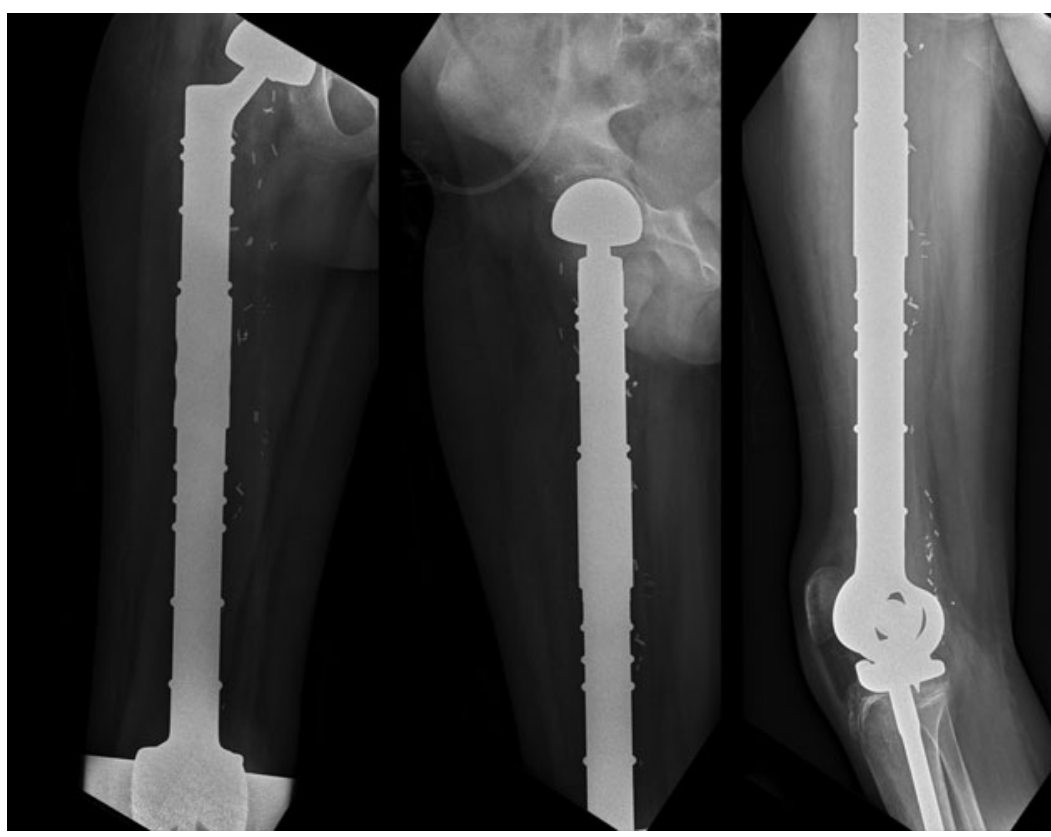

FIG. 1. Post-operative radiograph after Implantation of the total femur endoprosthesis.

modularity of the connecting element allowed optimum length adjustment of the rods at the trial reduction. Subsequently, the spacer was completely encased with antibiotic-loaded cement (Copal G + C, Heraeus-Sulzer, Germany) and implanted (Figs. 2, 3, and 4).

Post-operatively the antimicrobial treatment was continued with Rocephin and vancomycin according to the bacterial resistance. Under this therapy the patient's condition stabilized and the C-reactive protein (CRP) concentration normalized within nine days $(\mathrm{CRP}<10 \mathrm{mg} / \mathrm{L})$. The antibiotic therapy was continued for six weeks followed by a two-week antibiotic-free interval. During this period, the patient was afebrile and clinically stable.

Re-implantation of a total mega-endoprotheses was carried out two weeks after cessation of the antibiotic treatment and within eight days the CRP concentration normalized. The modular design also proved to be helpful in the explantation because for the dislocation no extensive release was required. After knocking off the cement from the interconnector, the spacer could be disconnected easily and removed in two parts. After removal of the spacer the re-implantation of a total hip socket and total femur mega-prosthesis was carried out.

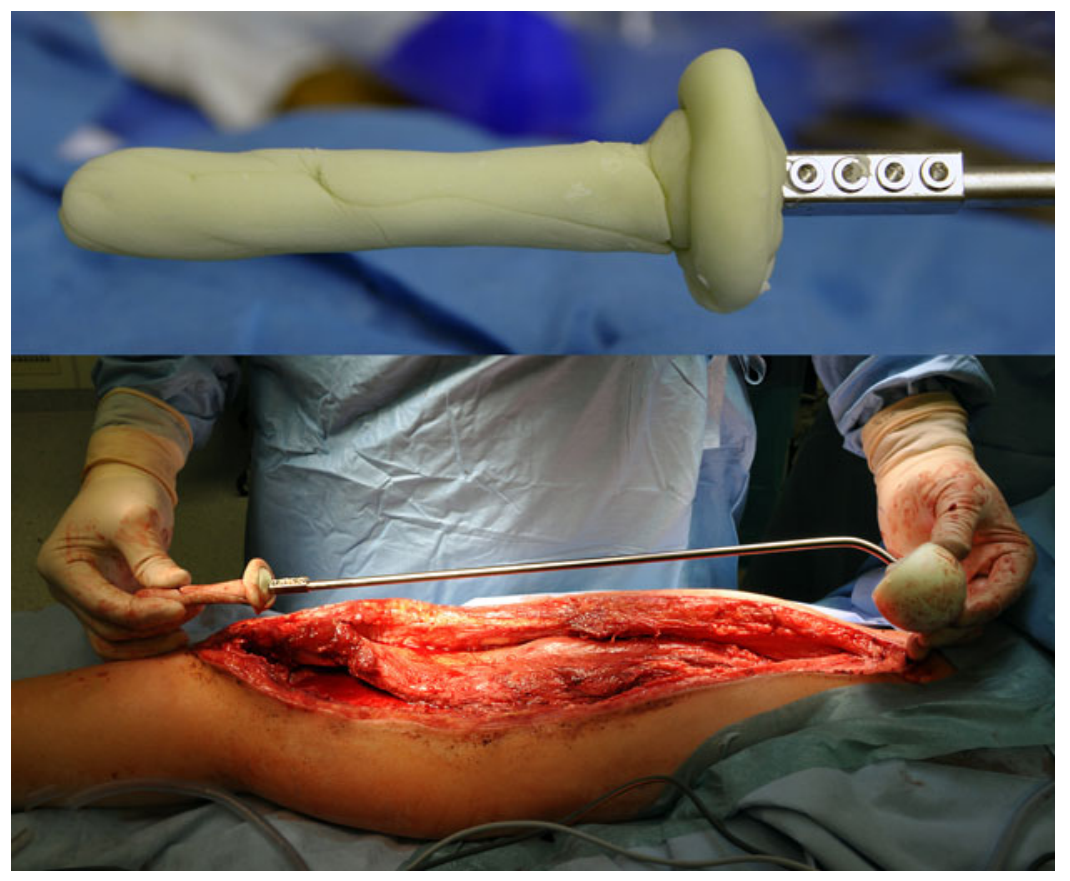

FIG. 2. The modularity of the mega-spacer allows individual adjustment to retain the correct length of the thigh. 


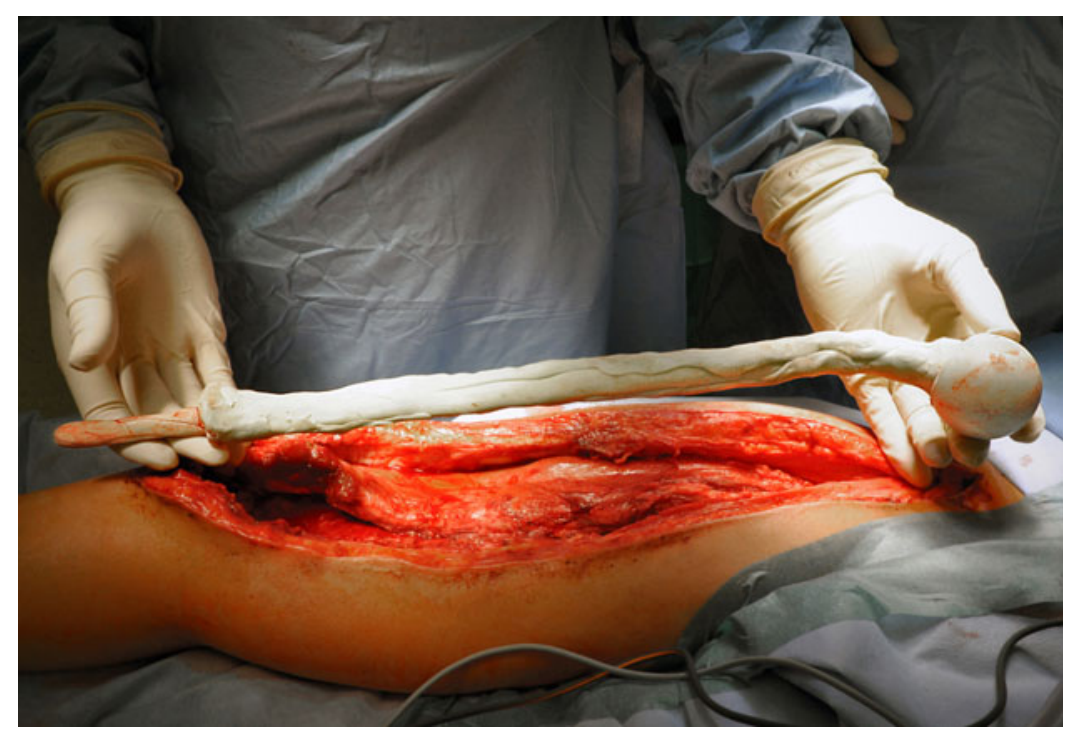

FIG. 3. In a second step the mega-spacer is completely encased with antibiotic-loaded cement.

At the time of re-implantation new microbial samples were taken. After receipt of the negative results we re-started a prophylactic therapy with Rocephin and vancomycin for a duration of 14 days.

At the last follow-up 30 months post-operatively the patient was afebrile and asymptomatic and showed nor- mal inflammatory markers without evidence of a new infection. The patient could walk without crutches, fully weight bearing, and had a range of motion of 90-degrees flexion and full knee extension. Because the proximal tibial and distal femoral ephiphysis were destroyed during the primary implantation an epiphysiodesis of the

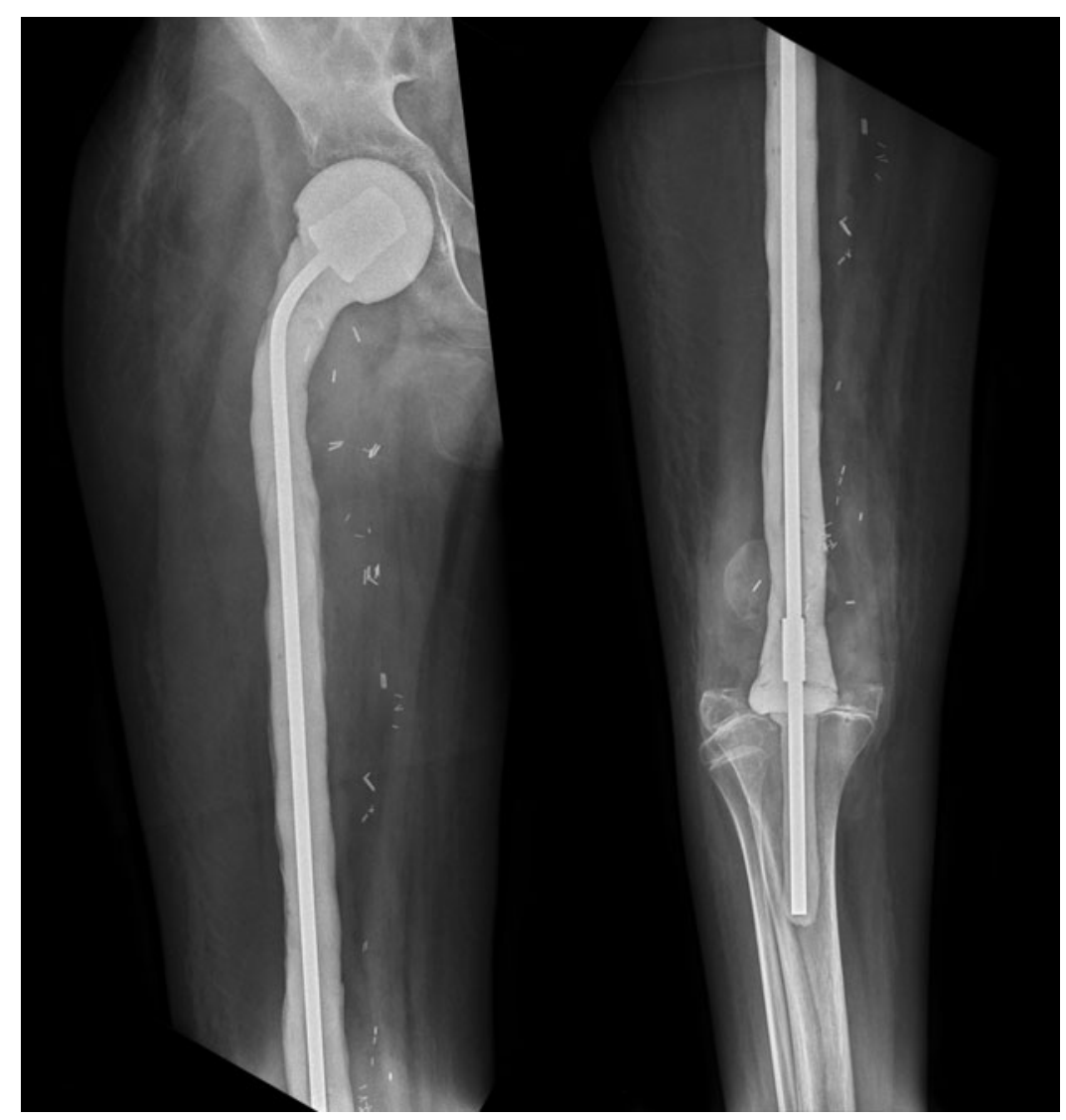

FIG. 4. Post-operative radiograph after implantation of the mega-spacer. 
contralateral side was performed 12 months after the revision surgery.

\section{Discussion}

Limb salvage procedures are complex operations with a corresponding risk of infection $[3,4]$. Because of the rarity and diversity of these cases there are no commercially available pre-fabricated solutions. Especially in pediatric orthopedics there is an additional challenge through the presence of growth plates. In our case the tibial epiphysis had to be resected because of infection. For this situation a mega-spacer had to meet the following requirements: (1) bridge two joints, namely hip and knee; (2) allow a good and safe mobilization including sitting; and (3) have a smooth round head for the preservation of acetabular bone stock after removal of acetabular cartilage. The length also had to be adjustable intra-operatively to prevent further loss of leg length.

Cassar Gheiti et al. [6] published two similarly complex cases treated successfully with a custom-made spacer. They used a polished hip stem combined with a femoral intramedullary nail that was coated with cement. A bipolar femoral head was used at the acetabulum. With this technique the acetabulum can also be preserved. However, an uncovered metallic surface increases the risk for new biofilm formation and hence failure of therapy.

Our method has several advantages in comparison to the method described by Cassar Gheiti et al. [6]. The use of a cemented head, as described in our case, leads to a fully cement-coated spacer. The fabrication of the described megaspacer does not require any expensive implants. The modularity allows to adjust for the correct length of the spacer and the position of the connector can be chosen freely depending on the individual situation (e.g., proximally or distally near the joint, diaphyseal, and so on).

Through its modularity the illustrated technique offers a good solution for individual adjustments for the preparation of interim prostheses especially for two-stage exchanges of mega-endoprosthesis. This optimum length can be adjusted easily. Furthermore, the explantation of the mega-spacer before replacement of a final prosthesis is simplified.

\section{Acknowledgments}

All procedures performed in studies involving human beings were in accordance with the ethical standards of the institutional and/or national research committee and with the 1964 Helsinki Declaration and its later amendments or comparable ethical standards. For this type of study formal consent is not required.

\section{Author Disclosure Statement}

The authors have no conflicts of interest to declare. No financial support was received for this study.

\section{References}

1. Plotz W, Rechl H, Burgkart R, et al. Limb salvage with tumor endoprostheses for malignant tumors of the knee. Clin Orthop Relat Res 2002;405:207-215.

2. Ham SJ, Schraffordt Koops H, Veth RP, et al. Limb salvage surgery for primary bone sarcoma of the lower extremities: Long-term consequences of endoprosthetic reconstructions. Ann Surg Oncol 1998;5:423-436.

3. Henderson ER, Groundland JS, Pala E, et al. Failure mode classification for tumor endoprostheses: Retrospective review of five institutions and a literature review. J Bone Joint Surg Am 2011;93:418-429.

4. Racano A, Pazionis T, Farrokhyar F, et al. High infection rate outcomes in long-bone tumor surgery with endoprosthetic reconstruction in adults: A systematic review. Clin Orthop Relat Res 2013;471:2017-2027.

5. Grimer RJ, Belthur M, Chandrasekar C, et al. Two-stage revision for infected endoprostheses used in tumor surgery. Clin Orthop Relat Res 2002;395:193-203.

6. Cassar Gheiti AJ, Baker JF, Brown TE, Mulhall KJ. Management of total femoral bone loss using a hybrid cement spacer surgical technique. J Arthroplasty 2013;28: 347-351.

7. Zimmerli W, Trampuz A, Ochsner PE. Prosthetic-joint infections. N Engl J Med 2004;351:1645-1654.

$$
\begin{array}{r}
\text { Address correspondence to: } \\
\text { Dr. Bernhard M. Speth } \\
\text { University Children's Hospital Basel } \\
\text { Department of Pediatric Orthopedic Surgery } \\
\text { Spitalstrasse } 33 \\
4056 \text { Basel } \\
\text { Switzerland }
\end{array}
$$

E-mail: Bernhard.Speth@gmail.com

\begin{tabular}{|l|}
\hline Abbreviations Used \\
CRP $=$ C-reactive protein \\
LSS $=$ limb salvage surgery
\end{tabular}

Cite this article as: Speth $\mathrm{BM}$, Krieg $\mathrm{AH}$, Hefti F, Nowakowski AM (2016) A custom-made modular megaspacer for the treatment of infection of a total femur prosthesis: A new surgical technique. Surgical Infections Case Reports 1:1, 149-152, DOI: 10.1089/crsi.2016.0045 